\title{
ESTRATÉGIA SAÚDE DA FAMÍLIA E A INTEGRALIDADE DO CUIDADO: PERCEPÇÃO DOS PROFISSIONAIS
}

\author{
FAMILY HEALTH STRATEGY AND CARE \\ INTEGRALITY: PROFESSIONALS' PERCEPTION
}

\section{ESTRATEGIA SALUD FAMILIAR Y LA INTEGRALIDAD DEL CUIDADO: PERCEPCIÓN DE PROFESIONALES}

\author{
Aline Pereira de Souza \\ Kátia Rezende ${ }^{2}$ \\ Maria José Marin ${ }^{3}$ \\ Silvia Tonhom ${ }^{4}$
}

\begin{abstract}
Como citar este artigo: Souza AP, Rezende K, Marin MJ, Tonhom S. Estratégia Saúde da Família e a integralidade do cuidado: percepção dos profissionais. Rev baiana enferm. 2020;34:e34935.

Objetivo: analisar a percepção dos profissionais das Equipes de Saúde da Família sobre a integralidade no cotidiano do cuidado. Método: estudo qualitativo realizado em quatro unidades da Estratégia Saúde. A coleta de dados foi realizada por meio de grupo focal com profissionais de diferentes categorias profissionais e contou com questões norteadoras sobre a compreensão de integralidade, como é desenvolvida, as potencialidades e as dificuldades em sua implementação. Os dados foram analisados por meio da análise de conteúdo, modalidade temática. Resultados: foram elaboradas duas temáticas: Integralidade na Atenção Básica revestida de complexidade e Desafios da integralidade devido à desarticulação intersetorial e dos níveis de atenção à saúde. Conclusão: dada a amplitude conceitual da integralidade, os profissionais das Equipes de Saúde da Família compreendem que esta se reveste de grande complexidade e enfrentam desafios importantes em sua implementação.
\end{abstract}

Descritores: Estratégia Saúde da Família. Sistema Único de Saúde. Integralidade em Saúde.

Objective: to analyze the perception of professionals from Family Health Teams about integrality in daily care. Method: qualitative study carried out in four Health Strategy units. Data collection was performed through a focus group with professionals from different professional categories and counted with guiding questions about the understanding of integrality, as it is developed, the potentialities and the difficulties in its implementation. Data were analyzed through thematic content analysis. Results: two themes emerged: Basic Care Integrality clothed with the complexity and Challenges of integrality due to intersectoral and health care levels disarticulation. Conclusion: given the conceptual breadth of integrality, the professionals from Family Health Teams understand that this is of great complexity and face important challenges in its implementation.

Descriptors: Family Health Strategy. Unified Health System. Integrality in Health.

\footnotetext{
Enfermeira. Especialista em Saúde Coletiva. Especialista em Enfermagem do Trabalho. Faculdade de Medicina de Marília. Marília, São Paulo, Brasil. aline.psouza_enfer@hotmail.com. https://orcid.org/0000-0003-4395-I482.

Enfermeira. Doutora em Enfermagem. Docente de Enfermagem na Faculdade de Medicina de Marília. Marília, São Paulo, Brasil. http://orcid.org/0000-0002-90222680.

3 Enfermeira. Doutora em Enfermagem. Docente de Enfermagem na Faculdade de Medicina de Marília. Marília, São Paulo, Brasil. http://orcid.org/0000-000 I-62 I 06941.

4 Enfermeira. Doutora em Educação. Docente de Enfermagem na Faculdade de Medicina de Marília. Marília, São Paulo, Brasil. http://orcid.org/0000-000 I-7522-286। .
} 
Objetivo: analizar la percepción de los profesionales de los Equipos de Salud Familiar en la integralidad en el cuidado diario. Método: estudio cualitativo realizado en cuatro unidades de Estrategia de Salud. La recolección de datos se realizó por medio de un grupo de enfoque con profesionales de diferentes categorías profesionales, utilizándose una guía de preguntas sobre la comprensión de la integralidad, como es desarrollada, las posibilidades y las dificultades en su aplicación. Los datos fueron analizados por medio de análisis de contenido temático. Resultados: se han elaborado dos temas: Integralidad en la Atención Básica vestida con la complejidad y Problemas de la integralidad debido a la desarticulación intersectorial y de los niveles de atención en salud. Conclusión: dada la amplitud conceptual de la integralidad, los profesionales de los Equipos de Salud Familiar comprenden que esta es de gran complejidad y enfrentan desafíos importantes en su aplicación.

Descriptores: Estrategia de Salud Familiar. Sistema Único de Salud. Integralidad en Salud.

\section{Introdução}

$\mathrm{Na}$ busca da implementação dos princípios e diretrizes do Sistema Único de Saúde (SUS), o da integralidade ganha muita relevância, visto que se trata de um "[...] conjunto articulado e contínuo das ações e serviços preventivos e curativos, individuais e coletivos, exigidos para cada caso em todos os níveis de complexidade do sistema ${ }^{(1: 2) ” . ~ D e s t a ~ f o r m a, ~ d e p r e e n d e-s e ~ o ~ s e u ~}$ carácter polissêmico e sua complexidade.

A integralidade revela-se como uma ação social resultante da interação democrática entre os sujeitos no cotidiano de suas práticas em saúde, transpondo a visão fragmentada do ser humano para uma compreensão integral, envolvendo aspectos biológicos, culturais e sociais do usuário nas dimensões individuais, familiares e coletivas ${ }^{(2)}$. Para atender a tal paradigma, o setor saúde tem como base estruturante a Estratégia Saúde da Família (ESF), que considera a importância da família em seu espaço social, abordando seu contexto socioeconômico e cultural, compreendendo que é nela que ocorrem as interações e os conflitos que influenciam diretamente o processo saúde-doença ${ }^{(2)}$. A ESF é considerada um cenário privilegiado de prática clínica ampliada para desenvolver a atenção e o acompanhamento continuado, capaz de viabilizar e potencializar esforços que impulsionem mudanças em direção à construção da integralidade ${ }^{(3)}$.

A efetivação do cuidado, na perspectiva da integralidade, ganha relevância essencialmente no cenário da Atenção Básica à saúde por constituir a porta de entrada aos demais níveis de atenção, na qual se articula todo o processo de conexão e acesso à rede de cuidados; o que nem sempre ocorre, devido à falta de comprometimento dos profissionais e dos gestores para promover a integralidade na prática, à concepção restrita do sentido da integralidade e à escassez de recursos estruturais para a sua operacionalização $^{(4)}$. Dessa forma, os profissionais de saúde devem percorrer o caminho em constante busca para ofertar cuidados de forma contínua, articulada e coordenada com vistas ao princípio da integralidade.

Ressalta-se que a integralidade do cuidado é construída na práxis dos diferentes profissionais inseridos nas distintas modalidades de cuidados em saúde, impondo como desafio a necessidade de uma nova forma de organização do processo de trabalho, bem como dos modos de agir e de interagir entre os distintos atores envolvidos. Compreende-se, assim, que essa construção implica romper com o saber fragmentado e descontextualizado e estabelecer uma prática de saúde pautada nas necessidades de saúde das pessoas, da família e da comunidade, as quais são influenciadas por valores, crenças e modos de vida, em conformidade com o contexto político, econômico e social ${ }^{(5)}$.

Dessa forma, tendo em vista o papel da integralidade na organização do SUS e na orientação dos serviços e das práticas do cotidiano em saúde, torna-se imprescindível que tal princípio direcione os profissionais de forma consciente e intencional, com vistas a atender às necessidades de saúde da população ${ }^{(6)}$.

Nesse contexto, em que o princípio da integralidade é fundamental quando se vislumbra melhoria da qualidade da atenção à saúde nos 
diferentes níveis de atenção, e a atenção básica constitui o eixo estruturante da rede de atenção, este estudo parte do seguinte questionamento: Quais são as potencialidades e os limites identificados por eles para realizarem promoção do cuidado na direção da integralidade?

O presente estudo tem como objetivo analisar a percepção dos profissionais das Equipes de Saúde da Família (EqSF) sobre a integralidade no cotidiano do cuidado.

\section{Método}

Estudo de abordagem qualitativa que tem como finalidade captar as representações, crenças, percepções e opiniões decorrentes das interpretações das pessoas sobre como vivem, sentem e pensam. Portanto, essa perspectiva possibilita desvelar processos sociais referentes a grupos específicos e permite a construção de novas abordagens e de novos conceitos no decorrer do processo de investigação ${ }^{(7)}$. No desenvolvimento do estudo seguiram-se as recomendações do Consolidated Criteria For Reporting Qualitative Research (COREQ) do EQUATOR Network, que tem como finalidade assegurar a reprodutibilidade da pesquisa em saúde ${ }^{(8)}$.

O estudo foi realizado em um município de médio porte do interior do estado de São Paulo que conta com uma população estimada de $238.882^{(9)}$. O serviço de atenção básica do muncípio, na ocasião da pesquisa, contava com 36 unidades de Saúde da Família (USF) e 12 Unidades Básicas de Saúde (UBS), as quais constituem a porta de entrada ao sistema de saúde.

O município organiza as unidades da atenção primária em saúde (APS) em quatro regiões (norte, sul, leste e oeste), e em cada uma delas existem tanto Unidades de Saúde da Família (USF), quanto Unidades Básicas de Saúde (UBS), considerando a diversidade do processo de viver e adoecer das pessoas desses territórios. Dessa forma, definiu-se como cenário de estudo a escolha de uma USF de cada região no intuito de representar essa heterogeneidade.

Fizeram parte da pesquisa os profissionais de quatro EqSF. A escolha de duas delas deu-se por conveniência, visto que se tratam das unidades em que uma das autoras desenvolveu as atividades durante o período da Residência Multiprofissional em Saúde Coletiva. Para a seleção das outras duas unidades, optou-se pela amostragem aleatória simples. Visando manter o anonimato das equipes selecionadas, elas receberam a numeração de um a quatro de acordo com a sequência do início da coleta de dados.

Foi convidado a participar desta pesquisa um profissional de cada categoria que compõe as equipes da ESF: Enfermeiro, Dentista, Médico, Auxiliar de Enfermagem, Agente Comunitário de Saúde (ACS), Auxiliar de Saúde Bucal (ASB), Auxiliar de Escrita e Auxiliar de Serviços Gerais. Para as categorias que contam com mais de um profissional, como os auxiliares de enfermagem e os ACS, os participantes foram definidos por amostragem aleatória simples.

No total, houve a participação de 29 profissionais, sendo oito da equipe um, oito da equipe dois, sete da equipe três e seis da equipe quatro. São quatro médicos, dois enfermeiros, quatro odontólogos, cinco auxiliares de enfermagem, quatro auxiliares de serviços bucais, três auxiliares de escrita e sete agentes comunitários de saúde. Dessa forma, contemplou-se a representação de cada categoria profissional que compõe as equipes da ESF, não havendo recusa dos profissionais convidados.

A coleta de dados foi realizada por meio de grupos focais, tendo em vista que dessa forma é possível apreender a complexidade do objeto, por meio da dinamicidade da interação entre os seus integrantes.

O grupo focal consiste de um diálogo em pequenos grupos homogêneos - de 6 a 12 participantes - a fim de obter informações, possibilitando a interação entre os participantes para fomentar consenso e ou divergências. A técnica deve ser operacionalizada mediante um roteiro de questões e o pesquisador deve ser capaz de garantir a participação e captar as perspectivas, os pensamentos, os conceitos de todos e de cada um ${ }^{(7)}$.

A proposição grupal para responderem às questões deve proporcionar descontração, gerar possibilidades contextualizadas pelo próprio 
grupo de estudo, de forma a facilitar a formação de novas ideias e possibilitar o estreito entendimento do tema no cotidiano dos envolvidos no processo ${ }^{(10)}$.

A coleta foi realizada nos meses de outubro a dezembro de 2017, nas respectivas unidades de trabalho, no horário em que ocorreram as reuniões de equipe, por se tratar de um momento em que as unidades ficam fechadas ao atendimento público, não interferindo no atendimento à população.

Antes de inicar os trabalhos, os participantes se apresentaram e, em seguida, uma das pesquisadoras apresentou a problemática, os objetivos, os cenários e os participantes do estudo. Os grupos focais foram conduzidos pela orientadora da investigação, docente da Famema, que possui o título de doutor em Enfermagem em Saúde Pública e conta com experiência na realização de grupo focal. Essa pesquisadora conduziu o grupo por meio das questões norteadoras, instigando a participação de todos. Esse trabalho contou com o envolvimento da residente, a qual realizou observações quando ao desenvolvimento do grupo, registrando-as.

O desenvolvimento dos grupos focais contou com as seguintes questões norteadoras: Qual sua compreensão a respeito do cuidado pautado na integralidade?; Como o cuidado integral é desenvolvido pela equipe?; Fale sobre as potencialidades/facilidades para promoção do cuidado pautado na integralidade; Fale sobre os limites/ dificuldades para promoção do cuidado pautado na integralidade.

A realização dos grupos contou com uma coordenadora, docente do Curso de Enfermagem e com experiência na área, além de uma observadora que realizou a gravação. Os diálogos, que tiveram duração média de 50 minutos, foram gravados e transcritos na íntegra. Os dados foram submetidos à Análise de Conteúdo, na modalidade temática, entendida como um conjunto de estratégias para extrair dos discursos diversificados seus sentidos e significados. Esse tipo de análise conduz à busca dos "núcleos de sentidos" inseridos em uma comunicação, confrontando-os com o objetivo do estudo e o referencial teórico ${ }^{(11)}$.
Nessa trajetória, após o trabalho de campo e a transcrição dos diálogos que emergiram dos grupos focais, os dados passaram pelas etapas de ordenamento e organização do mateiral empírico, seguidos da compreensão desse material, de forma a proporcionar valor, ênfase, espaço e tempo. Em seguida, deu-se o momento de transição entre o empírico e a elaboração teórica, ainda com a finalidade de apropriação ao máximo das informações obtidas, já em um esforço para ultrapassar o nível descrito do material. Após esse momento de impregnação do material, procede-se a elaboração das unidades de sentido e efetua-se a correlação com a teorização existente e, por fim, elabora-se o texto final ${ }^{(7)}$. A elaboração das temáticas foi feita inicialmente por duas pesquisadoras e, na sequência, as demais autoras fizeram a validação.

A interpretação dos resultados foi pautada na integralidade do cuidado em saúde, comprendida como um eixo prioritário da atual Política Nacional de Saúde ${ }^{(12)}$.

Esta pesquisa foi aprovada pelo Comitê de Ética em Pesquisa (CEP) da instituição proponente, de acordo com as Resoluções n. 466/2012 e 510/2016 do Conselho Nacional de Saúde que regula a pesquisa envolvendo seres humanos, mediante Parecer Consubstanciado do CEP n. 2.291.819 e CAEE n. 73033417.5.0000.5413. Os participantes que concordaram em participar do estudo assinaram o Termo de Consentimento Livre e Esclarecido (TCLE).

\section{Resultados}

No processamento da análise dos dados, foram identificados sete núcleos de sentido que foram agrupados em dois temas: Integralidade na Atenção na Básica revestida de complexidade e Desafios da integralidade devido à desarticulação intersetorial e aos níveis de atenção à saúde, conforme descritos a seguir:

\section{Integralidade na Atenção Básica revestida de complexidade}

Os núcleos de sentidos articulados a esse tema são: Desafios da compreensão das singularidades 
envolvidas na promoção da integralidade do cuidado; Formação profissional centrada no modelo biológico dificulta a promoção da integralidade do cuidado; A falta de comprometimento dos usuários com o cuidado em saúde dificulta a promoção da integralidade do cuidado; A falta de comprometimento dos usuários com o cuidado em saúde é decorrente do modelo médico centrado e da sua construção social.

Em relação à operacionalização do cuidado na direção da integralidade, emergiu entre as equipes a confirmação da complexidade de promover o cuidado, tendo em vista que as pessoas são diferentes, pois seus valores, desejos, expectativas foram construídos mediante diferentes condições de vida. A seguir, a fala que revela este núcleo de sentido:

Tem tanta coisa, "Olha você precisa tirar o cocô dessas galinhas, não pode deixar muitas galinhas em cima da mesa [...] 'Olha, ela é esposa do detento', dois filhos dela são viciados; aí você vai pra outra família, 'Olha, infelizmente a menina tem que se prostituir porque não tem como ganhar dinheiro' [...] uma filha que é casada com um rapaz que está preso, é traficante”. Então assim, o critério que ela usa para ser casada com traficante, cê acha que ela vai cuidar? Sabe assim, muito complexo. (ODONTO 3).

Aponta-se também, a formação profissional pautada no modelo biomédico hegemônico, o que dificulta a promoção da integralidade do cuidado.

[...] e até eu acho que dentro da equipe tem muito desconhecimento com relação à integralidade. Foi até bom ter abordado esse tema aqui. (MEDICINA 1).

Eu acho que uma das coisas que eu vejo que falta bastante até, é o olhar da questão social. A gente pensa no biológico, até na questão emocional, mas o social eu penso que é muito deixado de lado. (ENFERMAGEM 2).

[...] quando a equipe tenta também trabalhar a prevenção, apesar de que também tem aquela questão, todos os profissionais foram formados no modelo curativista, né, é uma questão real pra todos. (ENFERMAGEM 1).

A falta de comprometimento dos usuários com o cuidado em saúde dificulta a promoção da integralidade do cuidado, aspectos esses que emergiram das equipes:

[...] a educação dos usuários e a corresponsabilidade. Porque ninguém quer mexer no sal da comida, na dieta, ninguém quer fazer exercício, só quer que baixa a pressão naquela borinha ali [...] a gente vai atrás ou oferece, explica. Às vezes ele não tem o conbecimento devido, você esclarece pra ele tudo e ainda assim ele é relutante [...] move todo mundo pra resolver, solucionar o proble- ma. Aí quando tá tudo certinbo, tudo encaminhado, a pessoa não aceita ajuda, quer dizer, esse aí é o agravante, porque, todo mundo se envolve, cada um se mobiliza. (MEDICINA 1).

[...] não tem jeito, a gente faz, mas eles não mudam. Eles pedem ajuda, mas também não fazem nada para se ajudarem [...] Tem horas que você acha que é por falta até de conbecimento, é um contexto tão complexo [...] Então você não sabe se é "relaxo", porque a boca já está em uma situação precária, não tem orientação, não tem essa cultura de cuidar, e não tem as vezes noção de quanto custa, que é caro perder uma vaga dessas e aí não dão valor, uns não são isso, é que não tinha dinbeiro para ir. É muito complexo. (ODONTO 3).

É preciso considerar, entretanto, que ocorrem dificuldades nas mudanças de hábitos de vida, sendo necessário atentar para as singularidades dos sujeitos, bem como seu entorno e sua realidade socioeconômica.

Não adianta você falar pra alguém que ele precisa comer carne e verduras escuras todos os dias, quando ele está passando fome e não tem nem o arroz pra comer, entendeu? Não adianta você passar pra ela um remédio que vai custar $R \$ 120 R \$ 150$ reais, quando ele vive do programa de renda minima do governo. (ENFERMAGEM 2).

Os profissionais acreditam que a falta de comprometimento dos usuários com o cuidado em saúde acontece devido à questão cultural estabelecida por um modelo ainda hegemônico no Brasil, isto é, o modelo centrado nas ações médico-curativas, excessivamente especializado, de alto custo e baixa resolutividade, ou seja, um cuidado fragmentado e não centrado no sujeito.

O profissional indica o tratamento. Nem sempre ele dá continuidade ou ele saiu da crise e para o tratamento, "eu já sarei". Então tem que estar sempre atrás dele e tudo [...] sem contar que, às vezes, você pode até perder o vínculo [...] por aquela questão da cultura, é o curativo, o curativismo. [...] A questão do curativismo é muito intensa. Às vezes não só dos profissionais, mas da população também, a população tem essa questão e eles têm dificuldade de ouvir a equipe, quando a equipe tenta trabalhar a prevenção. (ACS 1).

\section{Desafios da integralidade devido à desarticulação intersetorial e aos níveis de atenção à saúde}

Os núcleos de sentidos articulados a esse tema são: Dificuldade de acesso ao nível de atenção secundária; Ineficácia do processo de referência e contra referência; Articulação com os demais setores é de responsabilidade da gestão municipal da saúde. 
De modo geral, denota-se entre os profissionais o desenvolvimento do cuidado integral. No entanto, evidencia-se que esse cuidado se restringe e se fragiliza no momento em que as necessidades de saúde ultrapassam os "muros" da unidade. Diante destas situações, considera-se que sua governabilidade é restrita e aponta-se para os limites do trabalho em rede.

\begin{abstract}
Tanto o setor secundário quanto o terciário estão na mesma situação, um caos se for pensar [...] tem especialidades de oftalmologia que nem tem na rede, tem que encaixar em outra especialidade [...] não atende realmente àquela necessidade identificada pelo serviço. Isso frustra um pouco, porque é a questão daquela rede de apoios que deveria ter [...] e aí a integralidade eu sinto que fica um pouco prejudicada. (ACS 1).

A dificuldade maior é dar continuidade no tratamento, porque a hora que chega aqui você tenta fazer tudo, mas a hora que depende de outras forças é uma dificuldade grande. Vamos supor, quando o paciente precisa de um exame, que é um exame muito caro, esse exame fica parado, ele demora um ano ou seis meses para fazer. E depende desse exame para dar continuidade no problema dela, então é uma dificuldade muito grande isso aí para nós, a gente sofre junto com o paciente. (ACS 4).
\end{abstract}

Destaca-se entre as equipes a não efetivação do processo de referência e contra referência, considerados ferramentas fundamentais para a integralidade da atenção à saúde.

[...] dificilmente a gente tem a contra referência, fica perdido esse acompanhamento. Ai com o trabalho da equipe a gente consegue resgatar a história com o paciente. Então, a tentativa de se fazer a integralidade, eu acho que fica fragmentada nesse sentido, quando você depende de outros níveis e de outras áreas também. (MEDICINA 4).

Aponta-se, ainda, que essa fragilidade no sistema pode gerar custos desnecessários:

Pessoa vai na UPA [Unidade de Pronto Atendimento] $e$ faz dois, três exames e daí manda para o bospital central, faz outro exame e manda para Santa Casa, e depois ele volta aqui sem nenbum resultado de exame, não tem essa comunicação eletrônica que é tão fácil [...] Aí tem que pedir pelo amor de Deus e a pessoa tem que voltar lá para pedir uma cópia [...] (MEDICINA 3).

[...] fica dificil a partir do momento em que sai daqui, nem sempre você consegue ter um respaldo. Depois que vai para média, alta complexidade é dificil mesmo você estar acompanhando assim certinho, sabe? Até porque eles não conseguem passar, e o profissional de lá as vezes também falha em mandar pra nós um comunicado, até uma carta de alta, você fica perdido. (ENFERMAGEM 4).

Sinaliza-se, entre as equipes, que muitas ações envolvem instâncias que se encontram "fora" do setor saúde, fugindo completamente de sua governabilidade, visualiza-se que compete ao apoio institucional estabelecer redes e elos com os demais setores.

O apoio tinha que fazer a ponte entre um setor e outro [...] falta de saneamento básico, lá em baixo mesmo na comunidade, não tem saneamento básico, uma coisa que ainda é muito grave no Brasil e aqui nós temos essa realidade [...] um povo muito carente. (ENFERMAGEM 4).

Às vezes você está com dificuldade pra conversar com outro serviço, vamos dizer assim, ofluxo não está funcionando direito [...] então isso seria um papel do apoio [gestão municipal], e ai quando a gente busca esse apoio, "Ah, liga lá você, fala lá com a fulana" sabe? Isso é muito difícil, porque a gente acaba muitas vezes até se indispondo com o profissional de um outro setor. (ENFERMAGEM 3).

\section{Discussão}

Considerando a complexidade de promover o cuidado na perspectiva da integralidade e tratando-se da APS, a construção da integralidade do cuidado é concebida na redefinição das práticas, para criar vínculos, acolhimento e autonomia que valorizem a singularidade dos sujeitos como pontos de partida para qualquer intervenção ${ }^{(2)}$.

Sugere-se que as práticas em saúde são orientadas mediante conceitos individuais, $\mathrm{O}$ que compromete a efetivação da mudança do modelo de atenção. São necessários estudos e debates que visem elaborar um arcabouço teórico para nortear as políticas públicas, as práticas dos trabalhadores das EqSF e os processos de formação e educação permanente ${ }^{(13) .}$

Ademais, afirma-se ${ }^{(14)}$ que o entendimento do conceito de saúde é primordial para a elaboração de ações estratégicas, interdisciplinares e intersetoriais, com ações de promoção, prevenção de riscos e agravos à saúde. Identifica-se uma estratégia bem sucedida desenvolvida em Portugal em relação à reforma da APS. Investimentos como ensaios e experiências piloto para ajuizar a viabilidade sobretudo no que tange à formação e à valorização de médicos voltados para os cuidados primários e a APS enquanto prioridade na política de Estado ${ }^{(15)}$.

Denota-se ${ }^{(3)}$ que, para promoção do cuidado na perspectiva da integralidade, é necessário o olhar para as questões sociais, visto que as situações de vida e saúde divergem em cada caso 
e para cada pessoa. As necessidades de saúde podem ser de caráter mais abrangente, incluindo os diversos níveis de atenção e/ou outros setores da sociedade.

Em geral, na literatura internacional, cuidados integrados têm sido definidos como o conjunto funcional de práticas de atenção, articulados em torno das necessidades de saúde de cada cidadão por meio da fixação da responsabilidade clínica em um profissional capaz de ter uma visão ampla do processo saúde/doença ${ }^{(16)}$.

Visualiza-se nas práticas de atenção à saúde, expressiva interferência do modelo biomédico hegemônico. A conformação de um dado modelo de atenção envolve valores que orientam a concepção de saúde e de direito à saúde, a qual é influenciada pelos conhecimentos acumulados e pelo paradigma hegemônico de ciência ${ }^{(17)}$.

Constatam dificuldades na implementação de um modelo que rompa com esse paradigma e, assim, sinaliza-se que, para a construção de um novo modelo, há que se considerar, o cotidiano das práticas e as necessidades de saúde das pessoas ${ }^{(17) .}$

Considerar as necessidades do sujeito para organização, planejamento e execução do trabalho da EqSF é uma estratégia de mudança do modelo de atenção à saúde. Hegemonicamente, as práticas são construídas com base na doença, foco no indivíduo e descontextualizada de sua realidade. Ao associar as necessidades singulares dos usuários ao contexto sociocultural no qual eles estão inseridos, a ESF rompe radicalmente com o modelo hospitalocêntrico de atenção à saúde ${ }^{(13)}$.

Estudo $^{(18)}$ destaca as fragilidades na comunicação entre os profissionais nos diversos níveis de atenção. Afirma-se que os mecanismos estudados - formulário de referência e contra referência, resumo de alta, telefone e bilhetes, protocolos do Ministério da Saúde e sessões clínicas conjuntas - são subutilizados e, quando usados, apresentam divergências entre níveis, sugerindo a compartimentalização da ferramenta e não seu compartilhamento. Esse cenário contribui para a fragmentação do cuidado, dificultando a implementação das políticas públicas ${ }^{(18)}$.
Nesse sentido, encontra-se que os pontos de atenção secundária e sistema de apoio atuam de forma fragmentada da atenção primária à saúde, devido a deficiências nos fluxos e comunicação ou ausência de informações entre os profissionais e/ou serviços, o que compromete a integralidade do cuidado ${ }^{(19)}$.

Autores $^{(14)}$ revelam grandes fragilidades na comunicação para articulação em rede, que se restringe a encaminhamentos sem oportunidade de diálogo com os profissionais dos diferentes serviços. Ademais, evidencia-se que, muitas vezes, só tomam conhecimento sobre o processo dos encaminhamentos e dos atendimentos realizados em outros serviços por meio dos próprios pacientes, corroborando o que se constata na presente pesquisa e em outras.

Sobre o acesso a serviços especializados, ressalta-se ${ }^{(20)}$ a tese de que a atenção de média complexidade é, na atualidade, o principal desafio do SUS. A sobrecarga financeira assumida pelos municípios, a carência de profissionais especialistas e a consequente escassez de oferta de consultas em várias especialidades, bem como a dependência do setor privado, contribuem para a magnitude do problema.

É fundamental o reconhecimento da importância da coordenação para efetivação do sistema de referência e contra referência. Ainda, evidencia-se a tensão verificada entre os médicos da atenção básica e da atenção especializada. Como hipótese dessa situação, elencam-se fatores relacionados ao mercado de trabalho, à formação acadêmica e aos valores que definem a representação social acerca do generalista e do especialista $^{(21)}$. Estes são desafios importantes e necessários de serem analisados e superados para que ocorra a articulação entre os diferentes níveis de atenção.

A implantação de prontuários únicos e informatizados em toda a rede de atenção representa uma contribuição importante para a articulação entre os diferentes serviços da rede de atenção, além da necessidade de criar espaços de encontros entre os profissionais que possibilite a construção de elo de confiança e compartilhamento 
de conhecimentos. Aponta-se, ainda, a importância de investimentos em educação continuada que considerem os diferentes perfis e as necessidades dos trabalhadores, com a realização de metodologias ativas, participativas e que estimulem o protagonismo entre esses atores ${ }^{(18)}$.

No âmbito da ESF, o trabalho deve ser capaz de articular-se de modo a promover ações intersetoriais e fortalecer vínculos com equipamentos sociais. As práticas devem considerar a associação entre desigualdade social e saúde. Destaca-se o elevado grau de complexidade que orienta esse trabalho, pois deve se fundamentar na determinação social do processo saúde-doença e a sua relação familiar e comunitária. Baseado nesse olhar, evidencia-se a necessidade de uma abordagem integral da população adscrita à ESF e usuária do SUS e do estabelecimento de parcerias intersetoriais para o cuidado em saúde ${ }^{(13)}$.

Assinalam-se duas premissas acerca da ação intersetorial para a promoção da saúde. A primeira é de caráter político e propõe que a intersetorialidade permita a busca de intervenções mais integrais, e a segunda é de natureza técnica, pois a integração de diversos setores possibilita que as diferenças entre eles possam ser empregadas produtivamente para enfrentar os problemas sociais $^{(22)}$.

Estudo ${ }^{(17: 1876)}$ cita que "As perspectivas teóricas e políticas de implementação de um novo modelo de atenção à saúde no Brasil são desafios que necessitam ser assimilados no cotidiano dos serviços de saúde, pelos profissionais de saúde, usuários e suas instâncias de controle social e pelos gestores da saúde."

O presente estudo tem como limitação o fato de abordar um momento e um cenário específico, entretanto, proporciona informações que contribuem para reflexões sobre o modelo de cuidado existente e aquele desejado.

\section{Conclusão}

O estudo analisou a concepção dos trabalhadores das Equipes de Saúde da Família de um município do interior paulista acerca da promoção do cuidado na perspectiva da integralidade.
Prepondera entre as equipes a complexidade de se operacionalizar o cuidado sob a perspectiva da integralidade, considerando que as pessoas são diferentes, visto que seus valores, desejos, expectativas foram construídos com base em distintas condições de vida.

Evidenciam limites na promoção do cuidado nessa direção, quais sejam: a formação profissional ainda está pautada no modelo biomédico, a não adesão e a falta de corresponsabilidade por parte dos usuários, além da desarticulação entre os diferentes níveis de atenção à saúde, e a inexistência da intersetorialidade.

Conclui-se que dada à amplitude conceitual da integralidade, os trabalhadores da atenção básica compreendem que esta se reveste de grande complexidade e enfrentam desafios importantes em sua implementação.

É preciso, então, considerar que para alcançar a integralidade no sistema de saúde existe a necessidade de os gestores municipais, estaduais e federais priorizarem este princípio, verificando no cotidiano de gestão do sistema se os pressupostos têm sido operacionalizados.

Acredita-se que a investigação tenha permitido elucidar a vivência da prática profissional, sendo possível afirmar que os esforços para a implementação do SUS vêm ocorrendo. No entanto, há necessidade de investimentos na formação de profissionais de saúde tanto no cenário da academia como dos serviços, bem como dos gestores, pois se reconhece que se trata de mudança paradigmática para alcançar o princípio da integralidade. Para tanto, faz-se necessário concretizar a estratégia de Educação Permanente em Saúde.

Evidencia-se a relevância da investigação para a prática de saúde em geral, e de enfermagem em particular, tendo em vista a importância do protagonismo do enfermeiro na APS, atuando na perspectiva da integralidade.

\section{Colaborações:}

1 - concepção, projeto, análise e interpretação dos dados: Aline Pereira de Souza e Kátia Rezende; 
2 - redação do artigo e revisão crítica relevante do conteúdo intelectual: Aline Pereira de Souza, Kátia Rezende, Maria José Marin e Silvia Tonhom;

3 - aprovação final da versão a ser publicada: Aline Pereira de Souza, Kátia Rezende, Maria José Marin e Silvia Tonhom.

\section{Referências}

1. Brasil. Lei no 8.080, de 19 de setembro de 1990 . Lei Orgânica da Saúde. Dispõe sobre as condições para a promoção, proteção e recuperação da saúde, a organização e o funcionamento dos serviços correspondentes e dá outras providências [Internet]. Brasília (DF); 1990 [cited 2017 Dec 2]. Available from: http://www.planalto.gov.br/ ccivil_03/leis/18080.htm

2. Motta LCS, Siqueira-Batista R. Estratégia Saúde da Família: Clínica e Crítica. Rev bras educ med. 2015;39(2):196-207. DOI: https://doi.org/10. 1590/1981-52712015v39n2e00912014

3. Viegas SMF, Penna CMM. The dimensions of the comprehensiveness on healthcare within the routine of the Family Health Strategy in the Jequitinhonha Valley, Minas Gerais, Brazil. Interface (Botucatu). 2015;19(55):1089-100. DOI: https://doi.org/10.1590/1807-57622014.0275

4. Heimann LS, Ibanhes LC, Boaretto RC, Castro IEN, Telesi Júnior E, Cortizo CT, et al. Atenção primária em saúde: um estudo multidimensional sobre os desafios e potencialidades na Região Metropolitana de São Paulo (SP, Brasil). Ciênc saúde coletiva. 2011;16(6):2877-87. DOI: https://doi.org/10.1590/ S1413-81232011000600025

5. Pires VMMM, Rodrigues VP, Nascimento MAA. Sentidos da integralidade do cuidado na saúde da família. Rev enferm UERJ [Internet]. 2010 [cited 2017 Oct 7];18(4):622-7. Available from: https://pesquisa.bvsalud.org/portal/resource/pt/ bde-19987

6. Nascimento LC, Arce VAR, Souza FDR. Concepções e práticas de integralidade em saúde no Núcleo de Apoio à Saúde da Família. Rev baiana saúde pública. 2018;42(1):26-40. DOI: https://doi. org/10.22278/2318-2660.2018.v42.n1.a2436

7. Minayo MCS. O Desafio do Conhecimento: pesquisa qualitativa em saúde. São Paulo: Hucitec; 2014.

8. Portugal AC, Santos CHS, Campos FM, Leoni L, França RR, Silva VS, et al. Artigo científico na área de saúde: diretrizes para sua elaboração e avaliação. Rev Ciênc Méd Biol. 2018;17(2)265-71. DOI: http://dx.doi.org/10.9771/cmbio.v17i2.25135

9. Instituto Brasileiro de Geografia e Estatística. Cidades e Estados [Internet]. Rio de Janeiro; 2019 [cited 2019 Dec 2]. Available from: https://www. ibge.gov.br/cidades-e-estados/sp/marilia.html

10. Ressel LB, Beck CLC, Gualda DMR, Hoffmann IC, Silva RM, Sehnem GD. O uso do grupo focal em pesquisa qualitativa. Texto Contexto Enferm. 2008;17(4):779-86. DOI: https://doi.org/10.1590/ S0104-07072008000400021

11. Minayo MCS. Análise qualitativa: teoria, passos e fidedignidade. Ciênc saúde coletiva. 2012;17(3):621-6. DOI: https://doi.org/10.1590/ S1413-81232012000300007

12. Pinheiro R, Mattos RA, organizadores. Construção Social da Demanda: direito à saúde, trabalho em equipe e participação em espaços públicos. Rio de Janeiro: CEPESC/UERJ:Abrasco; 2001.

13. Brito GEG, Mendes ACG, Santos Neto PM. O objeto de trabalho na Estratégia Saúde da Família. Interface (Botucatu). 2018;22(64):77-86. DOI: $10.1590 / 1807-57622016.0672$

14. Ferro LF, Silva EC, Zimmermann AB, Castanharo RCT, Oliveira FRL. Interdisciplinaridade e intersetorialidade na Estratégia Saúde da Família e no Núcleo de Apoio à Saúde da Família: Potencialidades e desafios. Mundo saúde. 2014;38(2):129-38. DOI: 10.15343/0104-7809. 20143802129138

15. Santos AM, Giovanella L, Botelho H, Sousa JC. Desafios Organizacionais para Fortalecimento da Atenção Primária à Saúde em Portugal. Rev bras educ med. 2015;39(3):359-69. DOI: https://doi. org/10.1590/1981-52712015v39n3e01542014

16. Smith G, Clarke D. Avaliando a eficácia de intervenções integradas: terminologia e abordagem. Med Clin North Am. 2006;90(4):533-48. DOI: https://doi.org/10.1016/j.mcna.2006.04.006

17. Fertonani HP, Pires DEP, Biff D, Scherer MDA. The health care model: Concepts and challenges for primary health care in Brazil. Ciênc saúde coletiva. 2015;20(6):1869-78. DOI: https://doi. org/10.1590/1413-81232015206.13272014

18. Oliveira CRF, Samico IC, Mendes MFM, Vargas I, Vázquez ML. Conhecimento e uso de mecanismos para articulação clínica entre níveis em duas redes de atenção à saúde de Pernambuco, Brasil. Cad Saúde Pública. 2019;35(4):e00119318. DOI: https:// doi.org/10.1590/0102-311x00119318 
19. Santos AM, Giovanella L. Gestão do cuidado integral: Estudo de caso em região de saúde da Bahia, Brasil. Cad Saúde Pública. 2016;32(3):1-15. DOI: https://doi.org/10.1590/0102-311X00172214

20. Silva CR, Carvalho BG, Cordoni Júnior L, Nunes EFPA. Difficulties in accessing services that are of medium complexity in small municipalities: a case study. Ciênc saúde coletiva. 2017;22(4):1109-20. DOI: https://doi. org/10.1590/1413-81232017224.27002016

21. Jesus RPFS, Santo ACGE, Mendes MFM, Samico IC. Percepção dos profissionais sobre a coordenação entre níveis de atenção à saúde em dois municípios pernambucanos de grande porte. Interface (Botucatu). 2018;22(65):423-34. DOI: https://doi. org/10.1590/1807-57622017.0026

22. Prado NMBL, Santos AM. Promoção da saúde na Atenção Primária à Saúde: sistematização de desafios e estratégias intersetoriais. Saúde debate. 2018;42(spe 1):37995. DOI: https://doi.org/ 10.1590/0103-11042018s126

Recebido: 20 de dezembro de 2019

Aprovado: 25 de março de 2020

Publicado: 30 de abril de 2020

The Revista Baiana de Enfermagem use the Creative Commons license - Attribuition -NonComercial 4.0 International. https://creativecommons.org/licenses/by-nc/4.0/

This article is an Open Access distributed under the terms of the Creative Commons (CC BY-NC).

This license lets others remix, adapt and create upon your work to non-commercial use, and although new works must give its due credit and can not be for comercial purposes, the users do not have to license such derivative works under the same terms. 